\title{
Anxiety and vestibular disorders
}

Volume 8 Issue 3 - 2017

\section{Communication}

The vestibular apparatus is located in the inner ear and its proper function is critical for postural control, maintaining equilibrium and spatial awareness. Current literature on anxiety and vestibular function indicate that there may be a relationship.

Anxiety disorders are the most frequent mental health concern. They are a set of related conditions, and each with single symptoms. However, all anxiety disorders have one thing in common: impact on day-to-day activities. ${ }^{1-3}$ Anxiety influences the ability to concentrate, sleep and carry out ordinary tasks.

Numerous studies have confirmed that anxiety and dizziness are interrelated. Dizziness can be associated with otologic, neurologic, medical, and psychiatric conditions. It is commonly occurs along with anxiety. ${ }^{4}$ If one is experiencing anxiety, dizziness can result. On the other hand dizziness can be anxiety-producing.

There is a multifaceted interface between vertigo and psychological distress. In some individuals, vertigo may lead to psychological distress, whereas the reverse appears to be true in others in that their psychological distress manifests as vertigo or dizziness. ${ }^{5}$ The mechanism that relations psychological distress and symptoms of dizziness or vertigo remains uncertain, but a number of researches have recommended that it is connected to the substantial overlap between the neuroanatomical regions and neurotransmitters concerned in the vestibular system and the pathways implicated in emotional states.

The dizziness that accompanies anxiety is normally described as a sense of light-headedness or wooziness. ${ }^{6}$ Environments as crowded malls or wide open spaces might cause loss of balance as symptom.

Often the anxiety and the dizziness should be treated mutually in order for better progress, once anxiety seems to be a meticulous problem in patients with acute and chronic vestibular disorders. ${ }^{7}$

A comprehensive multidisciplinary evaluation of dizziness holds great guarantee for more successfully diagnose and treatment. Cognitive-behavioral therapy and vestibular rehabilitation or drug therapy are also necessary, especially for the improvement of quality of life.

Clinicians must be attentive of the increased risk, and consequently the potential necessitate treating psychological disorders. Scales be supposed to be used as screen for anxiety in the early diagnosis and treatment, and psychologists/psychiatrist must be considered to assist, if necessary.

To conclude, anxiety and other psychiatric phenomenon must be considered when assessing patients with vestibular disorders.

\author{
Lilian Felipe \\ Fluminense Federal University Coordinator of Speech \\ Pathologist and Hearing Sciences, USA
}

Correspondence: Lilian Felipe Fluminense Federal University Rio de Janeiro Brazil Coordinator of Speech Pathologist and Hearing Sciences R Dr Silvio Henrique Braune 22 Nova Friburgo RJ 28625 650, Brazil, Email lilianfelipe@id.uff.br

Received: March 06, 2017 | Published: August 16, 2017

\section{Conflicts of interest}

Author declares there are no conflicts of interest.

\section{Funding}

None.

\section{References}

1. Best C, Eckhardt-Henn A, Diener G, et al. Interaction of somatoform and vestibular disorders. $J$ Neurol Neurosurg Psychiatry. 2006;77(5):658-664.

2. Best C, Eckhardt-Henn A, Tschan R, et al. Psychiatric morbidity and comorbidity in different vestibular vertigo syndromes. Results of a prospective longitudinal study over one year. $J$ Neurol. 2009;256(1):58-65.

3. Dieterich M, Staab JP, Brandt T. Functional (psychogenic) dizziness. Handb Clin Neurol. 2007;139:447-468.

4. Furman JM, Jacob RG. A clinical taxonomy of dizziness and anxiety in the otoneurological setting. J Anxiety Disord. 2001;15(1-2):9-26.

5. Horii A, Uno A, Kitahara T, et al. Effects of fluvoxamine on anxiety, depression, and subjective handicaps of chronic dizziness patients with or without neuro-otologic diseases. J Vestib Res. 2007;17(1):1-8.

6. Lahmann C, Henningsen P, Brandt T, et al. Psychiatric comorbidity and psychosocial impairment among patients with vertigo and dizziness. $J$ Neurol Neurosurg Psychiatry. 2015;86(3):302-308.

7. Staab JP. Chronic dizziness: the interface between psychiatry and neurootology. Curr Opin Neurol. 2006;19(1):41-48.

\section{Acknowledgments}

None. 